\title{
Usability Evaluation Considered Harmful (Some of the Time)
}

\author{
Saul Greenberg \\ Department of Computer Science \\ University of Calgary \\ Calgary, Alberta, T2N 1N4, Canada \\ saul.greenberg@ucalgary.ca
}

\author{
Bill Buxton \\ Principle Researcher \\ Microsoft Research \\ Redmond, WA, USA \\ bibuxton@microsoft.com
}

\begin{abstract}
Current practice in Human Computer Interaction as encouraged by educational institutes, academic review processes, and institutions with usability groups advocate usability evaluation as a critical part of every design process. This is for good reason: usability evaluation has a significant role to play when conditions warrant it. Yet evaluation can be ineffective and even harmful if naively done 'by rule' rather than 'by thought'. If done during early stage design, it can mute creative ideas that do not conform to current interface norms. If done to test radical innovations, the many interface issues that would likely arise from an immature technology can quash what could have been an inspired vision. If done to validate an academic prototype, it may incorrectly suggest a design's scientific worthiness rather than offer a meaningful critique of how it would be adopted and used in everyday practice. If done without regard to how cultures adopt technology over time, then today's reluctant reactions by users will forestall tomorrow's eager acceptance. The choice of evaluation methodology - if any - must arise from and be appropriate for the actual problem or research question under consideration.
\end{abstract}

\section{Author Keywords}

Usability testing, interface critiques, teaching usability.

\section{ACM Classification Keywords}

H5.2. Information interfaces and presentation (e.g., HCI): User Interfaces (Evaluation/Methodology).

In 1968, Dijkstra wrote 'Go To Statement Considered Harmful', a critique of existing programming practices that eventually led the programming community to adopt structured programming [8]. Since then, titles that include the phrase 'considered harmful' signal a critical essay that advocates change. This article is written in that vein.

Permission to make digital or hard copies of all or part of this work for personal or classroom use is granted without fee provided that copies are not made or distributed for profit or commercial advantage and that copies bear this notice and the full citation on the first page. To copy otherwise, or republish, to post on servers or to redistribute to lists, requires prior specific permission and/or a fee.

CHI 2008, April 5-10, 2008, Florence, Italy.

Copyright 2008 ACM 978-1-60558-011-1/08/04 ...\$5.00

\section{INTRODUCTION}

Usability evaluation is one of the major cornerstones of user interface design. This is for good reason. As Dix et al., remind us, such evaluation helps us "assess our designs and test our systems to ensure that they actually behave as we expect and meet the requirements of the user" [7]. This is typically done by using an evaluation method to measure or predict how effective, efficient and/or satisfied people would be when using the interface to perform one or more tasks. As commonly practiced, these usability evaluation methods range from laboratory-based user observations, controlled user studies, and/or inspection techniques $[7,22,1]$. The scope of this paper concerns these methods.

The purpose behind usability evaluation, regardless of the actual method, can vary considerably in different contexts. Within product groups, practitioners typically evaluate products under development for 'usability bugs', where developers are expected to correct the significant problems found (i.e., iterative development). Usability evaluation can also form part of an acceptance test, where human performance while using the system is measured quantitatively to see if it falls within an acceptable criteria (e.g., time to complete a task, error rate, relative satisfaction). Or if the team is considering purchasing one of two competing products, usability evaluation can determine which is better at certain things.

Within HCI research and academia, researchers employ usability evaluation to validate novel design ideas and systems, usually by showing that human performance or work practices are somehow improved when compared to some baseline set of metrics (e.g., other competing ideas), or that people can achieve a stated goal when using this system (e.g., performance measures, task completions), or that their processes and outcomes improve.

Clearly, usability evaluation is valuable for many situations, as it often helps validate both research ideas and products at varying stages in its lifecycle. Indeed, we (the authors) have advocated and practiced usability evaluation in both research and academia for many decades. We believe that the community should continue to evaluate usability for many - but not all - interface development situations. What we will argue is that there are some situations where 
usability evaluation can be considered harmful: we have to recognize these situations, and we should consider alternative methods instead of blindly following the usability evaluation doctrine. Usability evaluation, if wrongfully applied, can quash potentially valuable ideas early in the design process, incorrectly promote poor ideas, misdirect developers into solving minor vs. major problems, or ignore (or incorrectly suggest) how a design would be adopted and used in everyday practice.

This essay is written to help counterbalance what we too often perceive as an unquestioning adoption of the doctrine of usability evaluation by interface researchers and practitioners. Usability evaluation is not a universal panacea. It does not guarantee user-centered design. It will not always validate a research interface. It does not always lead to a scientific outcome. We will argue that:

the choice of evaluation methodology - if any - must arise from and be appropriate for the actual problem or research question under consideration.

We illustrate this problem in three ways. First, we describe one of the key problems: how the push for usability evaluation in education, academia, and industry has led to the incorrect belief that designs - no matter what stage of development they are in - must undergo some type of usability evaluation if they are to be considered part of a successful user-centered process. Second, we illustrate how problems can arise by describing a variety of situations where usability evaluation is considered harmful: (a) we argue that scientific evaluation methods do not necessarily imply science; (b) we argue that premature usability evaluation of early designs can eliminate promising ideas or the pursuit of multiple competing ideas; (c) we argue that traditional usability evaluation of inventions and innovations do not provide meaningful information about its cultural adoption over time. Third, we give general suggestions of what we can do about this. We close by pointing to others who have debated the merits of usability evaluation within the $\mathrm{CHI}$ context.

\section{THE HEAVY PUSH FOR USABILITY EVALUATION}

Usability evaluation is central to today's practice of HCI. In $\mathrm{HCI}$ education, it is a core component of what students are taught. In academia, validating designs through usability evaluation is

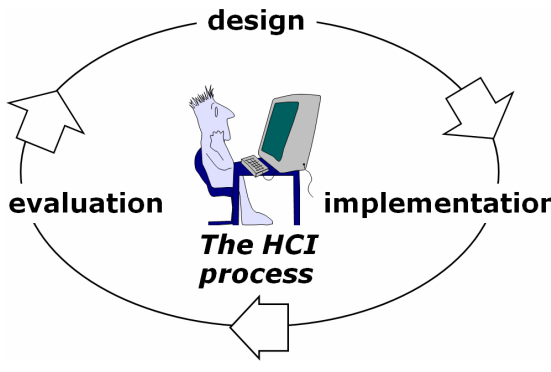

considered the de facto standard for submitted papers to our top conferences. In industry, interface specialists regard usability evaluation as a major component of their work practice.

\section{$\mathrm{HCl}$ Education}

The ACM SIGCHI Curriculum formally defines HCI as "a discipline concerned with the design, evaluation and implementation of interactive computing systems for human use..." [17, emphasis added].

The curriculum stresses the teaching of evaluation methodologies as one of its major modules. This has certainly been taken up in practice, although in a somewhat limited manner. While there are many evaluation methods, the typical undergraduate HCI course stresses usability evaluation - laboratory-based user observations, controlled studies, and /or inspection - as a key course component in both lectures and student projects [7,13]. Following the ACM Curriculum, the canonical development process drummed into students' heads is the iterative process of design, implement, evaluate, redesign, re-implement, reevaluate, and so on $[7,13,17]$. Because usability evaluation methodologies are easy to teach, learn, and examine (as compared to other 'harder' methods such as design, field studies, etc.), it has become perhaps the most concrete learning objective in a standard HCI course.

\section{CHI Academic Output}

Our key academic conferences such as ACM CHI, CSCW and even UIST strongly suggest that authors validate new designs of an interactive technology. For example, the ACM CHI 2008 Guide to Successful Submissions states:

\begin{abstract}
"does your contribution take the form of a design for a new interface, interaction technique or design tool? If so, you will probably want to demonstrate 'evaluation' validity, by subjecting your design to tests that demonstrate its effectiveness. [21]
\end{abstract}

The consequence is that the $\mathrm{CHI}$ academic culture generally accepts the doctrine that submitted papers on system design must include a usability evaluation - usually controlled experimentation or empirical usability testing - if it is to have a chance of success. Not only do authors believe this, but so do reviewers:

"Reviewers often cite problems with validity, rather than with the contribution per se, as the reason to reject a paper" [21]

Our own combined five-decades of experiences intermittently serving as Program Committee member, Associate Chair, Program Chair or even Conference Chair of these and other HCI conferences confirm that this ethic while sometimes challenged - is fundamental to how many papers are written and judged. Indeed, Barkhuus and Rode's analysis of ACM CHI papers published over the last 24 years found that the proportion of papers that include evaluation - particularly empirical evaluation - has increased substantially, to the point where almost all accepted papers have some evaluation component [1].

\section{Industry}

Over the last decade, industries are incorporating interface methodologies as part of their day-to-day development practice. This often includes the formation of an internal group of people dedicated to considering interface design as a first class citizen. These groups tend to specialize in usability evaluation. They may evaluate different design 
approaches, which in turn leads to a judicious weighing of the pros and cons of each design. They may test interfaces under iterative development - paper prototypes, running prototypes, implemented sub-systems - where they would produce a prioritized list of usability problems that could be rectified in the next design iteration. This emphasis on usability evaluation is most obvious when interface groups are composed mostly of human factors professionals trained in rigorous evaluation methodologies.

\section{Why this is a problem}

In education, academia and industry, usability evaluation has become a critical and necessary component in the design process. Usability evaluation is core because it is truly beneficial in many situations. The problem is that academics and practitioners often blindly apply usability evaluation to situations where - as we will argue in the following sections - it gives meaningless or trivial results, and can misdirect or even quash future design directions.

\section{USABILITY EVALUATION AS WEAK SCIENCE}

In this section, we emphasize concerns regarding how we as researchers do usability evaluations to contribute to our scientific knowledge. While we may use scientific methods to do our evaluation, this does not necessarily mean we are always doing effective science.

\section{The Method Forms the Research Question}

In the early days of CHI, a huge number of evaluation methods were developed for practitioners and academics to use. For example, John Gould's classic article How to Design Usable Systems is choc-full of pragmatic discount evaluation methodologies [12]. The mid-' 80 s to ' 90 s also saw many good methods developed and formalized by CHI researchers: quantitative, qualitative, analytical, informal, contextual and so on (e.g., [22]). The general idea was to give practitioners a methodology toolbox, where they could choose a method that would help them best answer the problem they were investigating in a cost-effective manner. Yet Barkhuus and Rode note a disturbing trend in the recent ACM CHI publications [1]: evaluations are dominated by quantitative empirical usability evaluations (about 70\%) followed by qualitative usability evaluations (about 25\%). As well, they report that papers about the evaluation methods themselves have almost disappeared. The implication is that ACM CHI now has a methodology bias, where certain kinds of usability evaluation methods are considered more 'correct' and thus acceptable than others.

The consequence is that people now likely generate 'research questions' that are amenable to a chosen method, rather than the other way around. That is, they choose a method perceived as 'favored' by review committees, and then find or fit a problem to match it. Our own anecdotal experiences confirm this: a common statement we hear is 'if we don't do a quantitative study, the chances of a paper getting in are small'. That is, researchers first choose the method (e.g., controlled study) and then concoct a problem that fits that method. Alternately, they may emphasize aspects of an existing problem that lends itself to that method, where that aspect may not be the most important one that should be considered. Similarly, we noticed methodological biases in reviews, where papers using nonempirical methodologies (e.g., case studies, field studies) are judged more stringently.

\section{Existence Proofs Instead of Risky Hypothesis Testing}

Designs implemented in research laboratories are often conceptual ideas usually intended to show an alternate way that something can be done. In these cases, the role of usability evaluation ideally validates that this alternate interface technique is better - hopefully much better - than the existing 'control' technique. Putting this into terms of hypothesis testing, the alternative (desired) hypothesis is in very general terms: "When performing a series of tasks, the use of the new technique leads to increased human performance when compared to the old technique".

What most researchers then try to do - often without being aware of it - is to create a situation favorable to the new technique. The implicit logic is that they should be able to demonstrate at least one case where the new technique performs better than the old technique; if they cannot, then this technique is likely not worth pursuing. In other words, the usability evaluation is an existence proof.

This seems like science, for hypothesis formation and testing are at the core of the scientific method. Yet it is, at best, weak science. The scientific method advocates risky hypothesis testing: the more the test tries to refute the hypothesis, the more powerful it is. If the hypothesis holds in spite of attempts to refute it, there is more validity in its claims [29, Ch. 9]. In contrast, the existence proof as used in HCI is confirmative hypothesis testing, for the evaluator is seeking confirmatory evidence. This safe test produces only weak validations of an interface technique. Indeed, it would be surprising if the researcher could not come up with a single scenario where the new technique would prove itself somehow 'better' than an existing technique.

\section{The Lack of Replication}

Rigorous science also demands replication, and the same should be true in CHI $[14,29]$. Replication serves several purposes. First, the HCI community should replicate usability evaluations to verify claimed results (in case of experimental flaws or fabrication). Second, the HCI community should replicate for more stringent and more risky hypothesis testing. While the original existence proof at least shows that an idea has some merit, follow-up tests are required to put bounds on it, i.e., to discover the limitations as well the strengths of the method $[14,29]$.

The problem is that replications are not highly valued in CHI. They are difficult to publish (unless they are controversial), and are rarely considered a strong result. This is in spite of the fact that the original study may have offered only suggestive results. Again, dipping into experiences on program committees, the typical referee 
response is 'it has been done before; therefore there is little value added'.

What exasperates the "it has done before" problem is that this reasoning is applied in a much more heavy-handed way to innovative technologies. For many people, the newer the idea and the less familiar they are with it, the more likely they are to see other's explorations into its variations, details and nuances as the same thing. That is, the granularity of distinction for the unknown is incredibly coarse. For example, most reviewers are well versed in graphical user interfaces, and often find evaluations of slight performance differences between (say) two types of menus as acceptable. However, reviewers considering an exploratory evaluation of (say) a new large interactive multi-touch surface, or of a tangible user interface almost inevitably produce the "it has been done before" review unless there is a grossly significant finding. Thus variation and replication in unknown areas must pass a higher bar if they are to be published.

All this leads to a dilemma in the $\mathrm{CHI}$ research culture. We demand validation as a pre-requisite for publication, yet these first evaluations are typically confirmatory and thus weak. We then rebuff publication or pursuit of replications, even though they deliberately challenge and test prior claims and are thus scientifically stronger.

\section{Objectivity vs. Subjectivity}

The attraction of quantitative empirical evaluations as our status quo (the $70 \%$ of our CHI papers as reported in [1]) is that it lets us escape the apparently subjective: instead of expressing opinions, we have methods that give us something that appears to be scientific and factual. Even our qualitative methods (the other $30 \%$ ) are based on the factual: they produce descriptions and observations that bind and direct the observer's interpretations. The challenge, however, is the converse. Our factual methods do not respect the subjective: they do not provide room for the experience of the advocate, much less their arguments or reflections or intuitions about a design.

The argument of objectivity over subjectivity has already been considered in other design disciplines, with perhaps the best discussion found in Snodgrass and Coyne's discussion of design evaluation in architecture by the experienced designer-as-assessor [25]:

[Design evaluation] is not haphazard because the assessor has acquired a tacit understanding of design value and how it is assessed, a complex set of tacit norms, processes, criteria and procedural rules, forming part of a practical know-how. From the time of their first 'crit', design students are absorbing design values and learning how the assessment process works; by the time they graduate, this learning has become tacit understanding, something that every practitioner implicitly understands more or less well. An absence of defined criteria and procedural rules does not, therefore, give free rein to merely individual responses, since these have already been structured within the framework of what is taken as significant and valid by the design community. An absence of objectivity does not result in uncontrolled license, since the assessor is conforming to unspoken rules that, more or less unconsciously, constrain interpretation and evaluation. If not so constrained, the assessor would not be a member of the hermeneutical community, and would therefore have no authority to act as an assessor. (p.123)

One way to recast this is to propose that the subjective arguments, opinions and reflections of experts should be considered just as legitimate as results derived from our more objective methods. Using a different calculus does not mean that one cannot obtain equally valid but different results. Our concern is that the narrowing of the calculus to essentially one methodological approach is negatively narrowing our view and our perspective, and therefore our potential contribution to $\mathrm{CHI}$.

Another way to recast this is that CHI's bias towards objective $v s$. subjective methods means it is stressing scientific contribution at the expense of design and engineering innovations. Yet depending on the discipline and the research question being asked, subjective methods may just as appropriate as objective ones.

A final thought before moving on. Science has one methodology, art and design have another. Are we surprised that art and design are remarkable for their creativity and innovation? While we pride our rigorous stance, we also bemoan the lack of design and innovation. Could there be a correlation between methodology and results?

\section{USABILITY EVALUATION AND EARLY DESIGNS}

We now turn to concerns regarding how we as practitioners do usability evaluations to validate designs. In particular, we focus on the early design stage where usability evaluation, if done prematurely, not only adds little value, but can quash what could have been promising design idea.

\section{Sketches vs. Prototypes}

Early designs are best considered as sketches. They illustrate the essence of an idea, but have many rough and/or undeveloped aspects to it. When an early design is displayed as a crude sketch, the team recognizes it as something to be worked on and developed further. Early designs are not limited to paper sketches: they can be implemented in a video, an interactive slide show, and as a running system as yet another way to explore the ideas behind a highly interactive system [3]. When systems are created as interactive sketches, they serve as a vehicle that helps a designer make vague ideas concrete, reflect on possible problems and uses, discover alternate new ideas and refine current ones [3,28,30,31].

The problem is that these working interactive sketches especially when their representation conveys a degree of refinement beyond their intended purpose - are often mistaken for prototypes, i.e., an approximation of a finished product. Indeed, the HCI literature rarely talks about working systems as a sketch, and instead elevates them to low / medium / high fidelity prototyping status [3], which people perceive as increasingly suggestive of the finished product. Yet this perception may be inappropriate, for prototypes are very different in purpose from a sketch. 


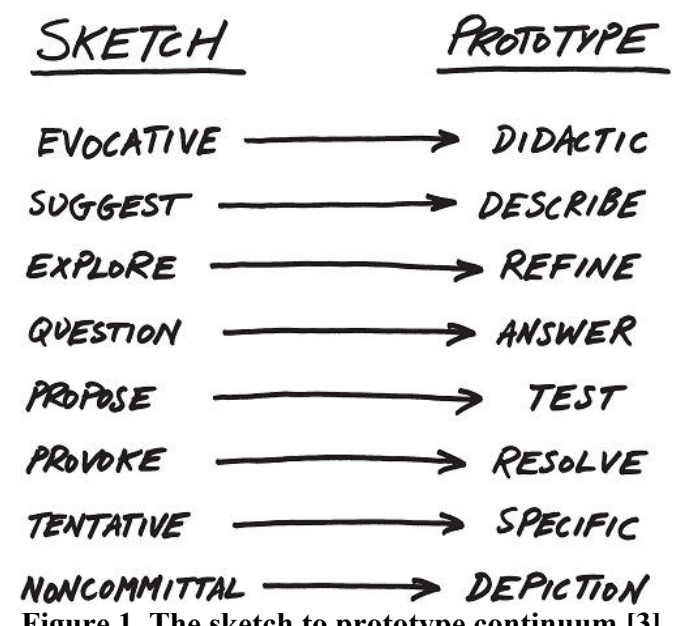

Figure 1. The sketch to prototype continuum [3].

Figure 1 illustrates this, where it defines the extremes between sketches and prototypes along several continua [3]. By definition, a sketch - even if implemented as an interactive system - is a roughed out design. It will have many holes, deficiencies, and undeveloped attributes. In contrast, a prototype aids idea evaluation, either by validating it with clients as they try it out, or through usability testing. Consequently, premature usability evaluation of the sketch as prototype could, unsurprisingly, find significant problems that could kill the design outright, especially if a novel design is compared to one that is more conservative. This has implications to both product developers and researchers.

Getting the Right Design vs. Getting the Design Right On the flip side, premature usability evaluation of sketches may also encourage developers to solve any of the problems seen by iterative refinement, as this is what the 'design, implement, evaluate' life cycle advocates. This leads to local hill climbing, where much effort is expended in 'Getting the Design Right' (Figure 2a). Unfortunately, evaluation of early sketches is often at the expense of considering and / or developing other, perhaps better, ideas.

A sketch typically illustrates only one of many possible designs and variations under consideration. Early design demands many idea sketches, reflecting on this multitude of competing ideas, and choosing the one(s) that appear the most promising (Figure $2 \mathrm{~b}$ ). The promising idea is then further varied and developed until it can serve as a testable prototype. That is, sketching is about 'Getting the Right Design' [31,3]. Only afterwards does one work on 'Getting the Design Right' of a particular idea through iterative testing and development. Thus sketching is akin to a heuristic that helps one move closer to the global maxima by circumventing the local hill climbing problem.

Usability evaluation methodologies favored by interface developers confound this problem. Most, like think-aloud observations, task centered walkthroughs, and heuristic evaluation, tend to focus on the negative: Where are the problems? What are the bugs? They do not inform us about the benefits. Yet ultimately, the underpinning of a

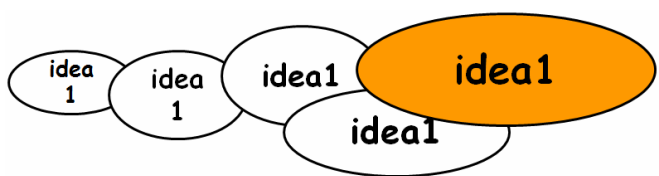

a) Getting the design right

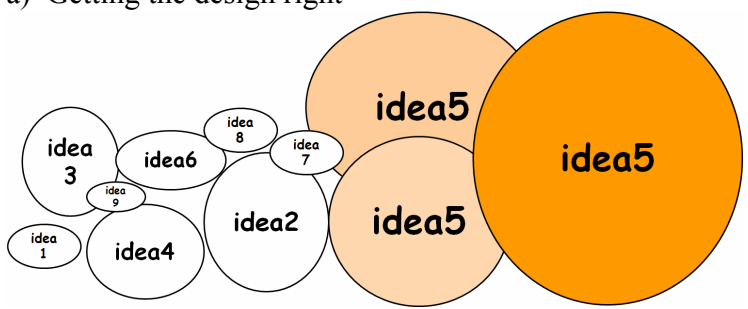

b) Generating designs, choosing the right design, and then getting the design right

Figure 2. Sketching first, iterative design and evaluation later meaningful evaluation is a cost/benefit analysis. The problem with our methodologies is that cost (problems) is easier to measure than benefit, and this focus on problems risks biasing decisions far too early in the process. Some of our early buggy designs may actually be the one that has the most potential for benefit in the long run, but we have no way of knowing this.

Similarly, CHI researchers favor usability evaluation methodologies - especially controlled experiments - as a way to validate a new technique by comparing its usability (as measured by some performance metric) to a longestablished technique. Again, this could quash a good idea simply because the design has not had a chance to evolve.

The net result is that we eliminate ideas too early, we consider far too few ideas at all, we converge on that which we can measure, which is almost always that which we are already familiar with. Our work degrades into a refinement of the known rather than innovation along new trajectories.

More generally, the ACM curriculum [17] suggests that design is an equal partner with evaluation. Yet the standard iterative cycle promoted within the $\mathrm{CHI}$ community is counter to traditional design practice. At issue is how the design/implement/test loop, if done naively, encourages the sequential evolution/refinement of ideas rather than the multiple parallel solutions that characterize most traditional design disciplines [3]. Usability evaluation should happen after multiple ideas are generated, critiqued, and considered. We include ourselves in this criticism, as we authored one of the first papers advocating an iterative approach to design [4], and promoted it in education [13].

\section{USABILITY EVALUATION, INNOVATION AND CULTURAL ADOPTION}

Invention and innovation is critical to CHI. In the small, they can significantly alter how we interact with a technology. In the large, new technologies and innovative interfaces can create a radical shift in the way we socialize, work, and play. Yet usability evaluation methods as done in research typically fails to consider the promise of novel engineering inventions and interface innovations. Similar to 
early designs, they are crude caricatures that suggest their usefulness, but are not yet developed enough to pass the test of usability. More importantly, usability evaluation tends to consider innovative products outside of their cultural context. Yet the reality is that it is the often unpredictable cultural uptake of the innovation that directs product evolution over time. These points are discussed below.

\section{Usable or Useful?}

Thomas Landauer eloquently argues in The Trouble with Computers that most computer systems are problematic because not only are they are too hard to use, but they do too little that is useful [19]. Most usability evaluation methodologies target the 'too hard to use' side of things: system designs are proven effective ('easy to use') when activities can be completed with minimal disruptive errors, efficient when tasks can be completed in reasonable time and effort, and satisfying when people are reasonably happy with the process and outcome $[16,19]$. The problem is that these measures do not indicate Landauer's second concern: design usefulness.

This distinction between usability and usefulness is not subtle. Indeed, the technological landscape is littered with unsold products that are highly usable, but totally useless. Conversely - and often to the chagrin of usability professionals - many product innovations succeed because they are very useful and/or fashionable; they sell even though they have quite serious usability problems. In practice, good usability in many successful products often happens after - not before - usefulness. A novel and useful innovation (even though it may be hard to use) is taken up by people, and then competition over time forces that innovation to evolve into something that is more usable. The World Wide Web is proof of this; many early web systems were abysmal but still highly used (e.g., airline reservations systems); usability came late in the game.

However, usefulness is a very difficult thing to evaluate, especially by the usability methodologies common in CHI. In most cases, it is often evaluated indirectly by determining (perhaps through a requirements analysis) what tasks are important to people, and using those tasks within scenarios to seed usability studies. If people are satisfied with how they do these tasks, then presumably the system will be both usable and useful. Yet determining usefulness of new designs is hard. In the Innovator's Dilemma, Christensen [5] argues that customers (and by extension designers who listen to them) often do not understand how new innovative technologies - especially those that seem to under-perform existing counterparts - can prove useful to them. It is left to 'upstart' companies to develop a technology: they find usage niches where it proves highly useful, and redesign it until it later (sometimes much later) becomes highly useful - and usable - in the broader cultural context.

Usability evaluation is predisposed to the world changing by gradual evolution; iterative refinement will produce more usable systems, but not radically new ones. It is ill disposed towards discontinuities as suggested by the Innovator's Dilemma, where sudden uptakes of useful or fashionable technologies by the community occur. If all we do is usability evaluation (which in turn favors iterative refinement), we will have a modest impact by making existing things better. What we will not do is have major impact by creating new innovations.

\section{Engineering Innovations and Cultural Adoption}

At the ACM UIST 2007 Panel on Evaluating Interface Systems Research, Scott Hudson distinguished the activities of 'Discovery' and 'Invention', and how they relate to evaluation methodologies. He said the goal of discovery is to find out facts of the world, whereas the goal of invention is to create and innovate new and useful things. Discovery, (normally the central task behind science), needs to meticulously detail facts so they may be used to craft theories that describe and/or predict phenomena. Rigorous and detailed evaluation is important in order to get the underlying facts upon which the theories rest correct. Typically we already understand the broad outlines or primary properties of the phenomena being studied, and make progress by looking at its very specific details (i.e., one or two variables) in order to distinguish between competing theories. In contrast, invention (normally the central task behind engineering and design) takes techniques - existing or new ones - that work in theory or in a lab setting, and extends them in often quite creative ways to work in the complexity of the real world. While rigorous evaluation may test a specific instant of that technique in a specific setting, concentrating on small details rarely predicts how variations of that technique will perform in other settings. The real world is complex and ever-changing: it is likely that 'big effects' are much more appropriate for study than small ones. Yet these 'big effects' are often much more difficult to evaluate with our classic usability evaluation methods.

Another way to say this is that usability evaluation, as practiced today, is appropriate for settings with well-known tasks and outcomes. Unfortunately, they fail to consider how novel engineering innovations and systems will evolve and be adopted by a culture over time. Let us consider a few historical examples to place this into context.

Marconi's wireless radio. In 1901, Guglielmo Marconi conducted the first trans-Atlantic test of wireless radio, where he transmitted in Morse code the three clicks making up the letter ' $s$ ' between the United Kingdom and Canada [32]. If considered as a usability test, it is less than impressive. First, the equipment setup was onerous: he even had to use balloons to lift the antenna as high as possible. Second, the sound quality was barely audible. He wrote

"I heard, faintly but distinctly, pip-pip-pip. I handed the phone to Kemp: "Can you hear anything?" I asked. 'Yes,' he said. 'The letter S.' He could hear it."

Yet his claim was controversial, with many scientists believing that the clicks were produced by random 
atmospheric noise mistaken for a signal. Whether or not this 'usability test' demonstrated the feasibility of wireless transmission, it is as interesting to consider how Marconi's vision of how radio would be used changed dramatically over time. Marconi is purported to have envisioned radio as a means for maritime communication between ships and shore; indeed, this was one of its first uses. He did not foresee what we take as commonplace: broadcast radio.

The automobile. Wireless radio is more of an infrastructure enabling end user system development, and perhaps outside the scope of usability tests. Instead, consider the automobile as a different kind of innovation. Cars were designed with end users in mind. Yet early ones were expensive, noisy, and unreliable. They demanded considerable expertise to maintain and drive them. They were initially impractical, as there was little in the way of infrastructure to permit regular travel. It is only after they were accepted by society that ideas of 'comfort', 'fashion' and 'ease of use' crept into automobile design, and even that happened only because one company saw it as a competitive advantage.

Bush's Memex. Let us now consider several great innovations in Computer Science in this context. In 1945, Vannevar Bush introduced the idea of cross-linked information in his seminal article As We May Think [2], which in turn inspired Hypertext and the World Wide Web. Bush described a system called 'Memex' based on linked microfilm records. Yet he never built it, let alone evaluated it. Bush's vision wasn't even correct: it was constrained to knowledge workers. He certainly never anticipated the use of linked records for what is now the mainstay of the web: social networking, e-commerce, pornography and gambling. Even if he had done a usability evaluation, it would have been based on tasks not considered central to today's culture. Still, there is no question that this was a valuable idea with profound influence on how people considered and eventually developed a new technology.

Sutherland's Sketchpad. In 1963, Ivan Sutherland produced Sketchpad, perhaps the most influential system in computer graphics and CAD [27]. Sketchpad was an impressive object-oriented graphics editor, where operators manipulated a plethora of physical controls (buttons, switches, knobs) in tandem with a light pen to create a drawing. No evaluation was done. Even if it were, it would probably have fared poorly due to the complexity of the controls and the poor quality display typical of this early technology.

Engelbart's NLS. In 1968, Douglas Engelbart gave what is arguably the most important system demonstration ever held in Computer Science [10]. He and his team showed off the capabilities of his NLS system. His vision as realized by NLS had a profound influence on graphical interfaces, hypertext, and computer supported cooperative work. Yet Engelbart's vision was about enhancing human intellect rather than ease of use. He believed that highly trained white collar people would use systems such as his to push the envelope of what is possible. Engelbart also failed to envision or predict the cultural adoption of his technologies by everyday folks for mundane purposes: he was too narrowly focused on productive office workers. Similar to Memex, even if Engelbart had done usability tests, they would have been based on a user audience and set of tasks that do not encompass today's culture.

Today's Compelling Ideas. To put this into today's perspective, we are now seeing many compelling ideas that suffer from limitations similar to the historic ones mentioned above. For example, consider the challenges of creating Ubiquitous Computing technologies (Ubicomp) for the home. Often, the technology required is too expensive (e.g., powerful tablet computers), the infrastructure is not in place (e.g., configuring hardware and wireless networks), the necessary information utilities are unavailable, or the system administration is too hard [9]. Even more important, the culture is not yet in place to exploit such technology: it is not a case of one person using Ubicomp, but of a critical mass of home inhabitants, relations, and friends adopting and using that technology. Only then does it become valuable. Again, cultural and technical readiness is needed before a system can be deemed 'successful'. How does one evaluate that except after the fact? Do usability evaluations of toy deployments really test much of interest? ${ }^{1}$

As a counterpoint, consider the many highly successful social systems now available on the web: Youtube, Facebook, Myspace, Instant Messaging, SMS, and so on. From an interface and functionality perspective, most of these systems can be considered quite primitive. Indeed, we can even criticize them for their poor usability. For example, Youtube does not allow one to save a viewed video. Thus one must be online to view a previously seen video; one must buffer the entire video even though only a fragment at the end is wanted; one must wait. Yet usability issues such as these are minor in terms of the way the culture has found an innovation useful, and how the culture adopted and evolved its use of such systems.

Discussion. There are several points to make about the above examples.

1. The innovations - whether ideas or working ones - were instances of a vision of what computer interfaces could be like. It is the vision - even if inaccurate - as well as the technology that was critical.

2. The visions foresaw the creation of a new culture of use, where people would fundamentally change what they would be able to do.

3. None of the inventions were immediately realized as products. Indeed, there was a significant lapse of time before the ideas within them were successfully incorporated into new products.

\footnotetext{
${ }^{1}$ They do, but as a way to inform design critique and reflection rather than testing. This interpretative use of testing is not normally considered part of our traditional process.
} 
4. The way innovations were taken up both in systems and by culture evolved considerably from the original vision of use: even our best visionaries had problems predicting how cultures would adopt technologies to their personal needs. Yet the vision was critical for stimulating work in the area.

5. Even if usability evaluations had occurred, they likely would have been meaningless. The underlying technology was immature, and any usability evaluation would highlight its limitations rather than its promise. As well, evaluations would have been based around user groups and task sets that would have little actual correspondence to how the technology would evolve in terms of its audience and actual uses.

Our argument is that using standard usability evaluation methods to validate innovations outside its culture of use is almost pointless (excepting for identifying slight usability problems). This leads to a dilemma: how can we create what could become culturally significant systems if we demand that the system be validated before a culture is formed around it? Indeed, this dilemma leads to a major frustration within CHI. We predominantly produce technology that is somewhat better than its antecedents, but these technologies rarely make it into the commercial world (although they may influence it somewhat). We also see innovative technologies that are highly successful but not developed by the CHI community, so we are left to evaluate its usability only after the fact. There is something wrong with this picture.

\section{WHAT TO DO}

We have argued that evaluation - while undeniably useful in many situations - can be harmful if applied inappropriately. There are several initiatives that we as a community can do to remedy this situation.

First, we need to recognize that usability evaluation is just one of the many methods that comprise our user-centered design toolkit, and that it should be used only when appropriate. As with any method, it should be brought into play only when the problem and the stage of UI development warrant it. There are many other aspects of user-centered design that are just as important: understanding requirements, considering cultural aspects, developing and showing clients design alternatives, affording new interface possibilities through technical innovations, and so on.

Second, we need to judge whether a usability evaluation at a particular point in our design cycle would produce anything meaningful. This means we need to continually reflect on our process, and consider the pros and cons. If the answer is 'no', then we should seek other methods to validate that stage of development. We outlined above several situations where this is likely: in very early design stages, in cases where usefulness overshadows usability, in instances where unpredictable cultural uptake dominates how an innovative system will actually be used.
Third, as a community we need to stop this blanket insistence on usability evaluation. This is not to say that we should accept hand-waving and slick demos as an alternative. As both an academic and practitioner community, we need to recognize that there are many other appropriate ways to validate one's work. Examples include a design rationale, a vision of what could be, expected scenarios of use, reflections, case studies, participatory critique, and so on. At a minimum, authors should critique the design: why things were done, what else was considered, what they learned, expected problems, how it fits in the broader context of both prior art and situated context, what is to be done next, and so on. These are all criteria that would be expected in any respected design school or firm. There is a rigour. There is a discipline. It is just not the same rigour and discipline that we currently encourage, teach, practice or accept. Academic paper submissions or product descriptions should be judged by the question being asked, the type of system or situation that is being described and whether the method the inventors used to argue their points are reasonable.

Fourth, when usability evaluations are appropriate for validating research designs, we should recognize that the formulatic way we do our evaluations (or judge them as publishable) often results in weak science. We need to change our methods to favor rigorous science. For really novel innovations, existence proofs are likely appropriate. For mainstream systems or modest variations of established ideas, we should likely favor risky hypothesis testing. We certainly should be doing more to help others replicate our results (e.g., by publishing data and/or making software available), and we should be more open-minded about accepting and encouraging replications in our literature.

Fifth, we should look to other disciplines to consider how they judge design worthiness. One example is the practice of design as taught in disciplines such as architecture and industrial design. Both employ the notion of a design studio: a place where people develop ideas into artifacts, and where surrounding people are expected to engage in discussion about these artifacts as they are being formed. These fields recognize that early designs are just 'sketches' that illustrate an idea in flux. Sketches are meant to change over time, and active discussion can influence how they change. Early evaluation is usually through the Design Critique (or 'Crit'). The designer presents the artifact to the group (typically a mix of senior and junior people), and explains why the design has unfolded the way it has. Members of the group respond: by articulating what they like and dislike about the idea, by challenging the designer's assumptions through a series of probes and questions, and by offering concrete suggestions of ways to improve the design. This is a reflective and highly interactive process: constructive criticisms and probing demands that designer and criticizers alike develop and share a deep understanding of the design idea and how it 
interacts within its context of use. ${ }^{2}$ Similarly, we need to understand methods that evaluate cultural aspects of designs. We are seeing some of this at ACM CSCW and UBICOMP, where ethnographic approaches are now considered vital if we are to understand how our technologies can be embedded within social and work groups, and within our physical environment. Other fields, such as Communication and Culture, have their own methods that may be appropriated for our use.

This list is incomplete, and we hope that others within CHI will add to it. Overall, what we are arguing for is a change in culture in how we do our research and practice, and in how we train our professionals, where we encompass a broad range of methods and approaches to how we create and validate our work.

\section{RELATED WORK}

We are not the first to raise cautions about the doctrine of evaluations in CHI. Henry Lieberman seeded the debate in his Tyranny of Evaluation, where he damns usability evaluation and the insistence that CHI places on it [20]. Shumin Zhai challenged his position by saying that in spite of the concerns, our evaluation methods are better than doing nothing at all [33]. Cockton continued the debate in 2007 [6], where he argued that the problem is not whether one should do evaluations, but that there is a lack of methods that are useful to various design stages, or to various practitioners (e.g., inventor $v s$. artist $v s$. designer $v s$. optimizer). Dan Olsen moderated a panel on evaluating interface systems research at UIST 2007. He raised concerns about how our expectations on measures of usability when evaluating interactive systems can create a usability trap, and offers alternate criterion for helping us judge systems [23].

Others raise concerns about the methods we use. Many standard textbooks offer the standard caveats to empirical testing, e.g., internal vs. external validity, statistical vs. practical significance, generalization, and so on [7]. Narrowing to the CHI arena, Stanley Dicks argues on the uses and misuses of usability testing in [26], while Barkhuus and Rode analyze the preponderance of usability evaluation in CHI and raise concerns about how such evaluations are now typically done [1]. Kaye and Sengers look at the evolution of evaluation in CHI: they stress the 'Damaged Merchandise' controversy that had practitioners from different fields challenging the usefulness of methods, particularly between advocates of discount methods vs. formal quantitative methods [18]. Pinelle and Gutwin analyzed evaluation in CSCW from 1990-1998 and found that almost $1 / 3$ of the systems were not formally evaluated, but perhaps more importantly that only about $1 / 4$ included evaluation in a real-world setting [24]. As CSCW systems

\footnotetext{
${ }^{2} \mathrm{http}: / /$ www.scottberkun.com/essays/essay $23 . \mathrm{htm}$
}

are often culturally situated, this raises serious questions about the evaluations that ignore real world context.

Of course, there are many people who argue that other nonevaluation methods can contribute to design. For example, Tohidi et. al. consider sketches as an effective way of getting reflective user feedback [30], while Buxton more generally considers the role of sketching in the design process [3]. On the cultural side, Gaver et. al. are developing methods that probe cultural reactions and technology uptake by niche cultures [11].

Finally, several splinter groups within the CHI umbrella arose in part as a reaction to evaluation expectations. ACM UIST emphasizes novel systems, interaction techniques, and algorithms - while evaluation is desired, it is not required if the design is inspiring and well argued (although they too are debating about how they are falling in the evaluation trap [23]). ACM CSCW and UBICOMP incorporate and nurture ethnography and qualitative methods as part of its methodology corpus, for both realized the importance of culture to understanding technological innovations. ACM DIS and DUX favors case studies that emphasize design, design rationale, and the design process.

\section{CONCLUSION}

We recapitulate our main message:

the choice of evaluation methodology - if any - must arise from and be appropriate for the actual problem or research question under consideration.

We should begin with the situation we are examining and the question we are trying to solve. We should choose a method that truly informs us about that situation or answers that question. More often than not, usability evaluation will be that method; this is why $\mathrm{CHI}$ has embraced it. Yet we should be open to other non-empirical methods - design critiques, design alternatives, case studies, cultural probes, reflection, design rationale - as being perhaps more appropriate for some of our situations.

It would be just as inappropriate to drop usability testing altogether in favor of the approaches that we are advocating. Zhai argues that usability evaluation is the best game in town [33], and we qualify by saying that this is true in many, but not all cases. For some cases, other methods are more appropriate. However, in all cases a combination of methods - from empirical to non-empirical to reflective - will likely help triangulate and enrich the discussion of a system's validity. It is just a matter of balance, but then, that is the true essence of evaluation anyhow!

Our concerns may appear novel to young CHI practitioners, but those who have been around will have heard them before and will likely have their own opinion. Regardless of who you are, consider how you can help enrich CHI. Join the debate. Change your development practices as a researcher and practitioner. Reconsider how you judge the papers while refereeing. Teach our new professionals that $\mathrm{HCI} \neq$ Usability Evaluation; it is far more than that. 


\section{ACKNOWLEDGMENTS}

This essay assimilates the many opinions, discussions, and arguments we have had or overheard between CHI colleagues over many, many years (mostly in bars, restaurants and hallway conversations). We thank them collectively. We also thank Beth Mynatt and our referees, whose comments helped us tighten our argument.

\section{REFERENCES}

1. Barkhuus, L., Rode, J. From Mice to Men - 24 Years of Evaluation in CHI. ACM CHI'07 - Alt.CHI. http://www.viktoria.se/altchi/ (2007).

2. Bush, V. As We May Think. Atlantic Monthly, (1945)

3. Buxton, B. Sketching User Experiences: Getting the Design Right and the Right Design. Morgan Kaufmann, (2007).

4. Buxton, W. \& Sniderman, R. Iteration in the Design of the Human-Computer Interface. Proc 13th. Meeting, Human Factors Assoc. of Canada, (1980), 72-81.

5. Christensen, C. The Innovator's Dilemma. Harper Business School Press, (1997).

6. Cockton, G. Make Evaluation Poverty History. ACM CHI'07 - Alt.CHI. http://www.viktoria.se/altchi/. (2007)

7. Dix, A., Finlay, J., Abowd, G. and Beale, R. Human Computer Interaction, $2^{\text {nd }}$ Edition, Prentice Hall, (1993)

8. Dijkstra, E. Go To Statement Considered Harmful. Comm. ACM 11(3): 147-148, (1968).

9. Edwards, W. and Grinter, R. At Home with Ubiquitous Computing: Seven Challenges. Proc UBICOMP. LNCS 2201, Springer-Verlag, (2001), 256-272.

10. Engelbart, D.C. and English, W.K. A Research Center for Augmenting Human Intellect. AFIPS Fall Joint Computer Conference, Vol. 33, (1968), 395-410.

11. Gaver, B., Dunne, T., and Pacenti, E. 1999. Design: Cultural probes. ACM Interactions 6:1, (1999), 21-29.

12. Gould, J.D. How to design usable systems. in R. Baecker, J. Grudin, W. Buxton and S. Greenberg (eds) Readings in Human Computer Interaction: Towards the Year 2000, Morgan-Kaufmann, (1996), 93-121.

13. Greenberg, S. Teaching Human Computer Interaction to Programmers. 3(4), ACM Interactions, (1996), 62-76.

14. Greenberg, S. 2008. Embedding a design studio course in a conventional Computer Science program. P. Kotzé. W. Wong, J. Jorge, A. Dix and P. Silva (eds): Creativity and HCI: From Experience to Design in Education Selected Contributions from HCIEd 2007.

15. Greenberg, S. and Thimbleby, H. The weak science of human-computer interaction. Proc CHI '92 Research Symposium on HCI (1992).

16. Gutwin, C. and Greenberg, S. The Mechanics of Collaboration: Developing Low Cost Usability Evaluation Methods for Shared Workspaces. Proc $9^{\text {th }}$ IEEE Int'l Workshop on Enabling Technologies:
Infrastructure for Collaborative Enterprises (WETICE'00). (2000).

17. Hewett, Baecker, Card, Carey, Gasen, Mantei, Perlman, Strong and Verplank (1996) ACM SIGCHI Curricula for Human-Computer Interaction. Last updated 2004-0603. http://sigchi.org/cdg/index.html

18. Kaye, J. and Sengers, P. The Evolution of Evaluation. ACM CHI'07 - Alt.CHI. http://www.viktoria.se/altchi/

19. Landauer, T. The Trouble with Computers: Usefulness, Usability, and Productivity. Cambridge, MA: MIT Press. (1995)

20. Lieberman, H. The Tyranny of Evaluation. web.media.mit.edu/ lieber/Misc/TyrannyEvaluation.ht $\mathrm{ml}$, ACM CHI Fringe, (2003).

21. Newman, W. CHI Guide to a Successful Archive Submission. http://www.chi2008.org/archiveGuide.html, (2008).

22. Nielsen, J. Usability Engineering. Morgan Kaufmann. (1993).

23. Olsen Jr., D. (2007). Evaluating User Interface Systems Research. Proc ACM UIST'07. ACM Press. 251-258.

24. Pinelle, D. and Gutwin, C. A Review of Groupware Evaluations. Proc $9^{\text {th }}$ IEEE Int'l Workshop on Enabling Technologies: Infrastructure for Collaborative Enterprises (WET-ICE'00). (2000). 86-91.

25. Snodgrass, A. \& Coyne, R. Interpretation in architecture: Design as a way of thinking. London: Routledge. (2006).

26. Stanley Dicks, R. Mis-Usability: On the Uses and Misuses of Usability Testing. Proc ACM SIGDOC, (2002)

27. Sutherland, I. Sketchpad: A man-machine graphical communication system. PhD Thesis, MIT, (1963).

28. Suwa and Tverskey. External representations contribute to the dynamic construction of ideas. In M. Hegarty, B. Meyer, and N. H. Narayanan (Eds.), Diagrams NY: Springer-Verlag, (2002), 341-343.

29. Thimbleby, H. User Interface Design. ACM Press Frontier Series, Addison-Wesley, (1990).

30. Tohidi, M., Buxton, W., Baecker, R., and Sellen, A. User Sketches: A Quick, Inexpensive, and Effective way to Elicit More Reflective User Feedback. Proc. NordiCHI (2006), 105-114.

31. Tohidi, M., Buxton, W., Baecker, R. and Sellen, A. Getting the Right Design and the Design Right: Testing Many is Better than One. Proc ACM CHI, (2006), 12431252.

32. Weightman, G. (2003) Signor Marconi’s Magic Box. Da Capo Press.

33. Zhai, S. Evaluation is the worst form of HCI research except all those other forms that have been tried, essay published at CHI Place, (2003). http://www.almaden.ibm.com/u/zhai/publications.html 